\title{
Palmprint Identification Integrating Left and Right by Local Discriminant Canonical Correlation Analysis
}

\author{
Shanwen Zhang*, Qinging Zhang, Hao Yuan \\ College of Electronic Information Engineering, Zhengzhou SIAS University, Zhengzhou 451150, China. \\ * Corresponding author. Tel.: +86 15091057958; email: wjdw716@163.com \\ Manuscript submitted February 10, 2019; accepted April 20, 2019. \\ doi: $10.17706 /$ jcp.14.9.580-589
}

\begin{abstract}
Palmprint based authentication has been investigated over 20 years, and many different problems related to palmprint recognition have been addressed, but it is still a challenging topic due to its importance, superiority, convenience and palmprints are often influenced by a lot of factors, such as illumination, viewing angle, noise, intentional fraud, wear, imperfection and pollution. Local discriminant canonical correlation analysis (LDCCA) is a well-known dimensional reduction algorithm to extract valuable information from multi-kinds of feature sets. Based on LDCCA, an authentication recognition method is proposed by using left and right palmprint. The method considers a combination of local properties and discrimination between different classes, including not only the correlations between sample pairs but also the correlations between samples and their local neighborhoods. Effective class separation is achieved by maximizing local withinclass correlations and minimizing local between-class correlations simultaneously. The experimental results on a public multimodal palmprint database CASIA validate the effectiveness of the proposed methods.
\end{abstract}

Key words: Palmprint recognition, palmprint pair identification, Canonical Correlation Analysis (CCA), Local Discriminant CCA (LDCCA).

\section{Introduction}

Today's electronically interconnected information society requires accurate automatic personal authentication schemes to authenticate a person's identity before giving an access to resources. Automatic authentication of humans is a very essential for law enforcement, public places such as airports, railway station and shopping complexes. Traditionally user authentication systems used for human recognition are passports, passwords, ration cards, ID cards, driving licenses etc. They are based on something one knows or something one has. The common disadvantage is that they are very susceptible for forgery, and can be lost or forgotten. Biometric based systems are possibly the best solution for human authentication [1], [2]. Today, people using advance technology of forgery and passwords hacking techniques to gain illegal access to services of a legitimate user. So, traditional approaches are no longer suitable for information society. Biometric pattern recognition is one of the trends used now days to identify human being by using their biometric traits [3], [4]. The automatic use of physiological or behavioral characteristics to determine or verify identity of individual's is regarded as biometrics. Fingerprints, Iris, Voice, Face, and palmprint are considered as physiological biometrics whereas voice and signature are behavioral biometrics [5], [6]. Among different sorts of biometric identifiers, palmprint identification, as a reliable human recognition method, has received an increasing attention and became an area of intense research during recent years, due to their high 
user acknowledgement and comfort. Palmprint is the image acquired of the palm region of the hand. It is either an online image taken by a scanner or CCD or offline image where the image is taken with ink and paper. The palmprint contains a lot of information for authentication, such as geometric, wrinkles (i.e., secondary lines), delta point, principal line, minutiae, ridge, etc., as shown in Fig. 1 [7].

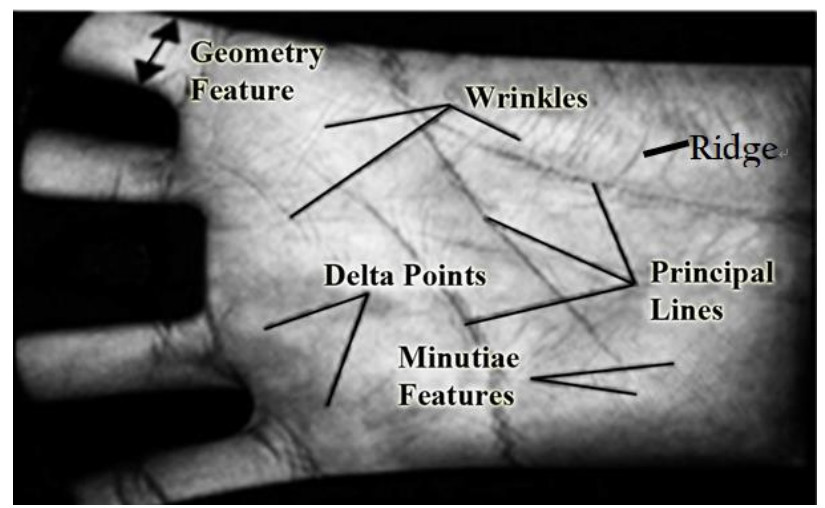

Fig. 1. Palmprint example.

Palmprint differs to a fingerprint in that it also contains other information such as texture, indents and marks which can be used to authenticate a person's identity. The problem with fingerprint is that, over a period of time, the fingerprint may loss or not properly readable so it cannot used for every age group of people. Palmprint can be used for forensic, criminal, and commercial applications. Palmprint, typically from the butt of the palm, are often found at crime scenes as the result of the offender's gloves slipping during the commission of the crime, and thus exposing part of the unprotected hand.

The previous palmprint recognition methods can be divided into two classes: extracting the principle lines and creases in the spatial domain and transforming the palmprint images into the frequency domain to obtain the energy distribution feature. In the first class, the lines and creases of a palm are sometimes difficult to extract directly from a given palmprint image with low resolution. The recognition rates and the computational efficiency are also not sufficient. In the second class, the abundant textural details of a palm are ignored and the extracted features are greatly affected by the lighting conditions. The problems with these two approaches suggest that new methods are required for palmprint recognition. Zhong et al. [7] provided an overview of current palmprint research, describing in particular capture devices, preprocessing, verification algorithms, palmprint related fusion, algorithms especially designed for real-time palmprint identification in large databases and measures for protecting palmprint systems and user privacy. Morales et al. [8] investigated the ridge pattern characteristics of the interdigital palm region for its usage in biometric identification, carried out an anatomical study of the interdigital area, leading to the establishment of five categories according to the distribution of the singularities and three regions of interest for biometrics. With the identified regions, they analyzed the matching performance of the interdigital palm biometrics and its combination with the conventional palmprint matching approaches and presents comparative experimental results using four competing feature extraction methods. This study has been carried out with two publicly available databases. Zhang et al. [9] developed a highly user-friendly device for capturing high-quality contactless palmprint images, and then established a large-scale palmprint image dataset with the developed device. To make the results fully reproducible, the collected dataset and the related source codes are publicly available at http://sse.tongji.edu.cn/linzhang/contactlesspalm/index.htm. In order to extract more effective palmprint feature, Feng et al. [10] proposed a method for feature extraction of palmprint for palmprint recognition, which could improve the efficiency of identification.

Feature extraction is one of most basic problems in the research of palmprint recognition. Extracting 
effective palmprint feature is the crucial problem in the field of palmprint recognition. There was a research focus on how to select the feature. The lacking of main orientation for palmprint recognition system will lead to an incorrect feature extraction and matching. The single biometric system may be inadequate for passive authentication either because of noise in data samples or because of unavailability of a sample at a given time. Mouad and Gaikwad [11] presented an overview of a current multimodal biometrics research based on fingerprint and palmprint, including the pervious study for each modal separately and its fusion technique with another biometric modal. The basic biometric based human identification method consists of four stages: collecting biometrics data, preprocessing including the enhancement and ROI segmentation, features extracted from the preprocessed data, and matching the extracted feature with the template in the database. In order to overcome the limitation of the single biometric and improve the security and reliability of the identification system, many multiple biometric modalities have been used. Lin et al. [12] proposed a combination recognition method of palmprint and palm vein based on gray surface matching. Kadri et al. [13] proposed a human identification method by combining palmprint and iris in order to construct an efficient multibiometric recognition system based on matching score level fusion and by using the technique of sum of score. This system tries to improve the recognition results of single biometric systems. The features of Iris and palmprint are extracted using Log Gabor filter. The Hamming Distance is used for matching of Iris or palmprint feature vector. Leng et al. [14] extracted the features of left and right palmprints with twodimensional discrete cosine transform (2DDCT) to constitute a dual-source space, and normalized the extracted features in dual-source space to avoid the disturbance caused by the coefficients with large absolute values. The experiments on contactless palmprint database confirm that multi-source palmprint fusion systems benefit of palmprint recognition and the advantages of biometric fusion systems. Many traditional palmprint recognition methods are mostly based on acquisition devices with contact, which may affect their user friendliness. Charfi et al. [15] proposed a toucheless palmprint identification method based on Scale Invariant Feature Transform (SIFT) descriptors and sparse representation method to extract palmprint features of left and right palms. The fusion features are used generate the final identity of a person at rank level using Support Vector Machines (SVM) classifier and probability distribution.

Canonical correlation analysis (CCA) is a well-known multivariate analysis method to evaluate the correlations between two sets of multi-modal variables. It is a linear dimensionality reduction method. However, there are many nonlinear relationships between features in practice. Locality preserving CCA (LPCCA) is proposed to incorporate local structure information into CCA for solving nonlinear dimensionality reduction problems [16]. As CCA, LPCCA only concerns the relationship between the sample pairs without utilizing the class information of samples that is essential for classification. Local discriminant CCA (LDCCA) introduces the class information of samples to classical CCA and considers the local correlations of the withinclass sets and the between-class sets [17], [18]. Different from CCA and LPCCA, LDCCA considers not only the correlations between the sample pairs but also the correlations between samples and their local neighborhoods.

Inspired by the development of multimodal biometric identification, a left and right palmprint biometric recognition method is proposed based on LDCCA.

The rest of the paper is organized as follows. Section 2 introduces LDCCA. Section 3, a biometric recognition method is proposed integrating left and right palmprint by LDCCA. Experimental results and analysis are provided in Section 4, and conclusion is given in Section 5.

\section{Local Discriminant CCA (LDCCA)}

CCA is a classical linear multi-view dimensionality reduction method to extract the useful information from 
multi-view feature sets. Suppose two different feature sets of $n$ samples: $X=\left[x_{1}, x_{2}, \ldots, x_{n}\right] \in R^{p \times n}$ and $Y=\left[y_{1}, y_{2}, \ldots, y_{n}\right] \in R^{a \times n}$, CCA aims to find two projection matrices $w_{x} \in R^{p}$ and $w_{y} \in R^{q}$ to maximize the correlation coefficient between $X$ and $Y$ by solving the following objective function of CCA,

$$
\max _{w_{x}, w_{y}} \frac{E\left\lfloor w_{x}^{T} x y^{T} w_{y}\right\rfloor}{\sqrt{E\left[w_{x}^{T} x x^{T} w_{x}\right]\left[w_{y}^{T} y y^{T} w_{y}\right]}}=\frac{w_{x}^{T} C_{x y} w_{y}}{\sqrt{w_{x}^{T} C_{x x} w_{x} \cdot w_{y}^{T} C_{y y} w_{y}}}
$$

where $E$ represents expectation, $C_{x x}=X X^{T}, C_{y y}=Y Y^{T}$ are the auto-covariance of $X$ and $Y$, respectively, and $C_{x y}=X Y^{T}$ is the cross covariance of $X$ and $Y$.

In order to improve the discriminant ability of CCA in classification tasks, LDCCA makes use of the discriminative information to maximize the correlations between intra-class samples, maximize the margins between inter-class samples, and project the multi-view features into a common low-dimensionality subspace such that the correlation of the projected vectors is maximized, meanwhile, preserve the local data structure, thus LDCCA achieves better performance. Similar to CCA, the objective function of local discriminant (LDCCA) is denoted as follows,

$$
\max _{w_{x}, w_{y}} \frac{w_{x}^{T} S_{x y} w_{y}}{\sqrt{w_{x}^{T} S_{x x} w_{x} \cdot w_{y}^{T} S_{y y} w_{y}}}
$$

where $S_{x x}=X X^{T}, S_{y y}=Y Y^{T}$ are the covariance of $X$ and $Y$, respectively, $S_{x y}=S_{w}-\lambda S_{b}$ is the cross covariance of $X$ and $Y, \lambda$ is an adjustment factor to make a trade-off between $S_{w}$ and $S_{b}$, and $S_{w}$ and $S_{b}$ are local within-class covariance matrix and local between-class covariance matrix, defined respectively as follows,

$$
\begin{aligned}
& S_{w}=\sum_{i=1}^{n} \sum_{x_{j} \in N^{w}}^{n}\left(x_{i}\right) \cap y_{j} \in N^{w}\left(y_{i}\right) \\
& \left.S_{b}=\sum_{i=1}^{n} \sum_{x_{j} \in N^{b}\left(x_{i}\right) \cap y_{j} \in N^{b}\left(y_{i}\right)}\left(x_{i}^{T} y_{j}^{T}+x_{j} y_{i}^{T}\right) y_{i}^{T}\right)
\end{aligned}
$$

where $N^{w}\left(x_{i}\right)$ and $N^{b}\left(x_{i}\right)$ are $k$ nearest neighborhoods of the within-class and between-class of $x_{i}, N^{w}\left(y_{i}\right)$ and $N^{b}\left(y_{i}\right)$ are $k$ nearest neighborhoods of the within-class and between-class of $y_{i}$.

Comparing Eqs. (1) with (2), it is found that the standard CCA optimization problem is slightly modified so that the cross-covariance matrix (between the two views) $C_{x y}$ in Eq. (1) is replaced by a term $S_{x y}$ in Eq. (2) which takes class information into account. It is defined as the sum of local within class covariance matrices penalized by the sum of local between class covariance matrices. It implies that samples nearby in the original space should be close together in the feature space. The idea of locality is defined in terms of $\mathrm{k}$ nearest neighborhood (k-NN). Eqs. (1) and (2) can be obtained by computing a generalized engen-value decomposition problem of the Lagrange multiplier method.

\section{Palmprint Recognition Based on LDCCA}

From the above analysis, we proposed a left and right palmprint recognition method based on LDCCA. Its steps is summarized as follows. 
Input: $n$ left and right palmprint pairs from $n$ training samples: $X=\left[x_{1}, x_{2}, \ldots, x_{n}\right] \in R^{p \times n}$ and $Y=\left[y_{1}, y_{2}, \ldots, y_{n}\right] \in R^{q \times n}$, three parameters $k, \lambda$ and $d$, and a left and right palmprint pair $(x, y)$ of a test sample.

Output: The label of the test sample.

Step 1: Compute local covariance matrices $S_{w}$ and $S_{b}$ according to Eq.(3), and get covariance matrices $S_{x x}=X X^{T}, S_{y y}=Y Y^{T}$ and $S_{x y}=S_{w}-\lambda S_{b}$;

Step 2: Compute matrix $H=\left(S_{x x}\right)^{-1 / 2} S_{x y}\left(S_{y y}\right)^{-1 / 2}$;

Step 4: Perform SVD decomposition $H=U D V^{T}$;

Step 5: Choose $\left[U_{1}, U_{2}, \ldots, U_{d}\right],\left[V_{1}, V_{2}, \ldots, V_{d}\right], d<r$;

Step 6: Obtain $W_{x}=\left(S_{x x}\right)^{-1 / 2}\left[U_{1}, U_{2}, \ldots, U_{d}\right], W_{y}=\left(S_{y y}\right)^{-1 / 2}\left[V_{1}, V_{2}, \ldots, V_{d}\right]$;

Step 7: Project feature vector pair $(x, y)$ into a low dimensional fusion feature vector, $V=\left(\begin{array}{l}W_{x}^{T} x \\ W_{y}^{T} y\end{array}\right)$, where $V$ is used for classification by any classifier.

Step: Classify the feature vector $V$ of the test sample by a classifier.

\section{Experiments and Analysis}

In this section, we validate the proposed palmprint recognition method on a real public palmprint dataset, namely CASIA multi-spectral palmprint image database V1.0 (i.e., CASIA for short), and compare it with the typical state-of-the-art palmprint identification methods, such as combining shape analysis and texture pattern (SATP) [19], using scale invariant feature transform features (SIFT) [20], combining left and right palmprints (LRP) [21], and using LPCCA [16]. Among these algorithms, SATP and SIFT are two single modal biometrics based on multi-feature integration using all palmprints, while LRP and LPCCA are two multibiometrics combining the left and right palmprints, K-nearest neighbor (KNN) classifier is adopted to recognize palmprints. Note that all the algorithms adopt principle components analysis (PCA) to reduce the data dimensionality. All the experiments are carried out on a PC with double-core Intel(R) i5-3470 $3.2 \mathrm{GHz}$ CPU, RAM 8.00GB, and Windows 7.0 operating system and MATLAB 7.10.0.

\subsection{Dataset Preparation}

CASIA Palmprint Image Database (or CASIA-Palmprint for short) contains 5,502 palmprint images captured from 312 subjects, and the number of palms per individual is from 16 to 34 , in which each left palm is matched with each right palm of every individual (http://biometrics.idealtest.org/). Fig. 2 is the Selfdeveloped multi-spectral imaging device. For each subject, palmprint images from both left and right palms were collected by the Chinese Academy of Sciences' Institute of Automation (CASIA)" and a reference to "CASIA Palmprint Database" should be included. All palmprint images are 8 bit gray-level JPEG files by our

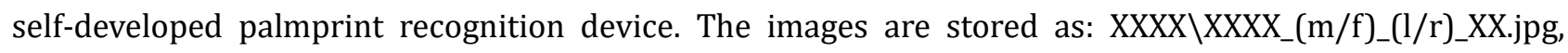
where XXXX: the unique identifier of people, ranging from 0000 to 0312; (m/f): gender ' $\mathrm{m}$ ' denotes male and ' $\mathrm{f}$ ' female; (l/r): type of palm ' l' denotes left palm and ' $r$ ' denotes right palm; XX: the index of image with the same type of palm. Mostly it ranges between 1 and 15. In the dataset, several palms are misplaced and some palms are distorted with poor quality. Some typical palmprint examples are shown in Fig. 3 and 4. From Fig. 3 and 4 , it is found that all images have more obvious palm movements, distortions and blurring, and have a certain degree of variations of hand postures, which may increase the intra-class difference, and meanwhile increase the diversity of intra-class samples and simulation practical use. So CASIA is a challenging dataset for palmprint identification algorithm validation. 


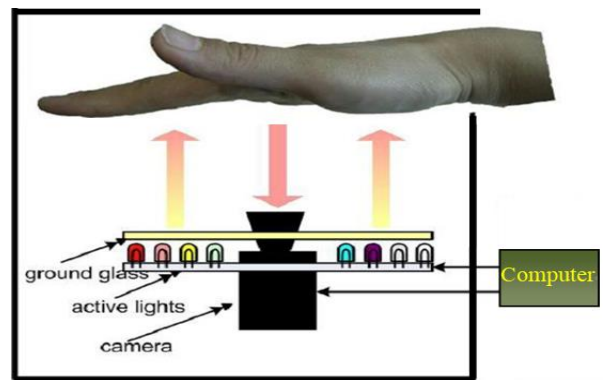

Fig. 2. Self-developed multi-spectral imaging device of CASIA.
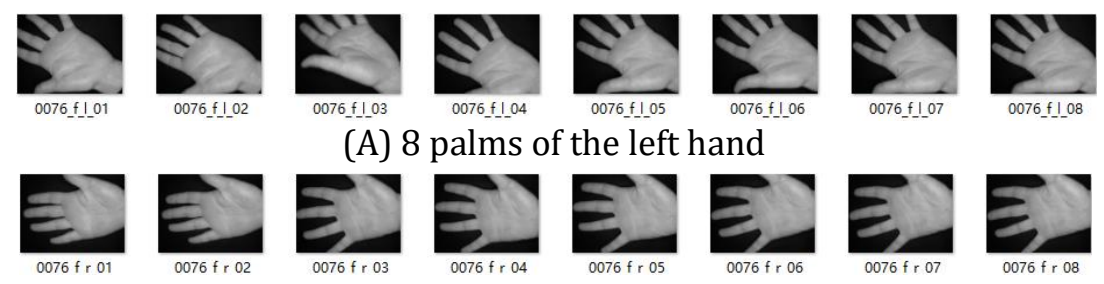

(B) 8 palms of the right hand

Fig. 3. Typical palms of the 76th individual in CASIA.
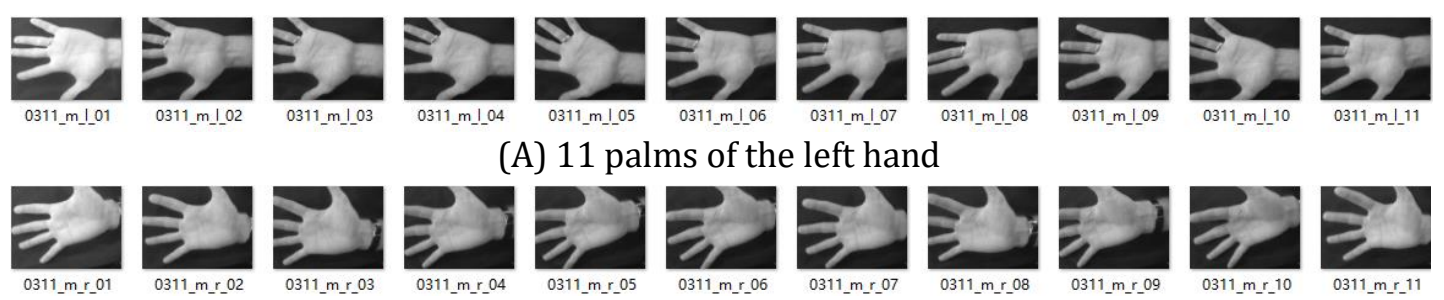

(A) 11 palms of the left hand
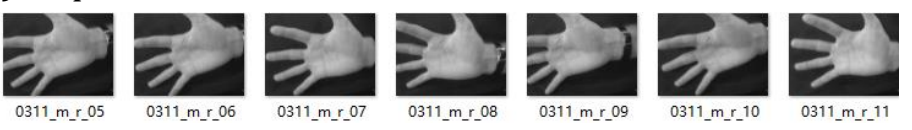

(B) 11 palms of the right hand

Fig. 4. Typical palms of the 311th individual in CASIA.

The preprocessing is done to align the various palms and segment the region of interest (ROI) for feature extraction. The most common steps of preprocessing include Gaussian smoothing, binary processing, edge detecting by boundary tracing algorithm, and ROI segmenting. The detailed processing procedure are explained in [6]-[9], the main process as shown in Fig. 5. Firstly compute the key point between the two gaps of fingers to get $\mathrm{Y}$-axis and then establish coordinate, where $\mathrm{P}_{1}$ and $\mathrm{P}_{2}$ are two detecting points. Finally segment a sub image (i.e., ROI) of a fixed size based on coordinate system. The segmented ROI is scaled with size of $64 \times 64$ pixels, as given in Fig. $5 \mathrm{D}$, which is called palmprint image, namely palmprint. After preprocessing, all scaled ROI images form a real palmprint set for individual identification, which is also denoted as CASIA dataset in the following experiments.

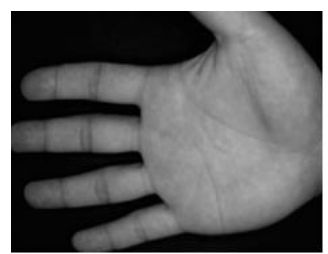

(A) Original palm

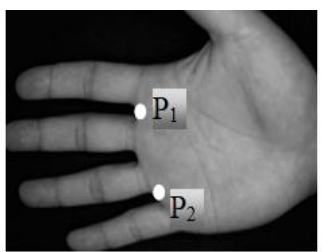

(B) Key point

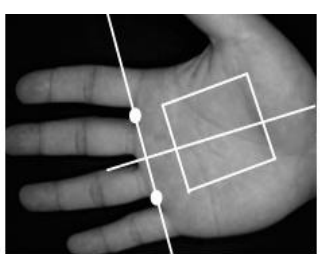

(C) Coordinate axis

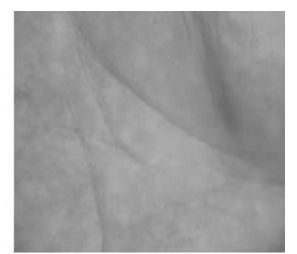

(D) Palmprint

Fig. 5. Palmprint preprocessing.

\subsection{Experiment Results}

At first, in CASIA, left and right palmprints of each individual are matched to a left-right palmprint pair 
subset, and then we can obtain 3600 pairs from 312 individuals. After preprocessed, each palmprint is represented as a point in 1-D vector space for classification by concatenating each column of a 2-D palmprint. That is to say, a typical image of size $64 \times 64$ with 256 gray levels per pixel is described as a point in 4096dimensional vector in image space. No further preprocessing is done. Secondly, PCA is often applied to reduce the vector dimensionality by selecting all principal components corresponding to the non-zero eigenvalues. Thirdly, low-dimensional fusion classifying features are extracted from the left-right palmprint pairs by LDCCA. Finally, individuals are identified based on the extracted features using 1-NN classifier for its simplicity. In order to control the relative contributions of $S_{w}$ and $S_{b}$ in LDCCA, a balancing factor $\lambda$ is introduced, which is optimized by searching in the range $\lambda \in[0.001,0.01,0.1,1,10,100]$. In the following experiments, 5 -fold-cross-validation is performed to find the optimal values of the above three parameters $k, \lambda$ and $d$ in their respective ranges. However, it is noted that if we jointly optimize three parameters together, there will produce a huge search space, thus finding the optimal values will be very time-consuming. So we optimize those parameters independently, i.e. in a 'one-by-one' manner.

In order to test the effect in term of the reduction dimensions in the reduced subspace on the recognition rate, Fig. 6 shows the identification accuracy of LDCCA versus the reduction dimensionalities. From Fig. 6, it is found that, in the beginning, the recognition rates increase with the dimensions become large, while larger dimension will not lead to higher recognition rate after LDCCA achieves the best results.

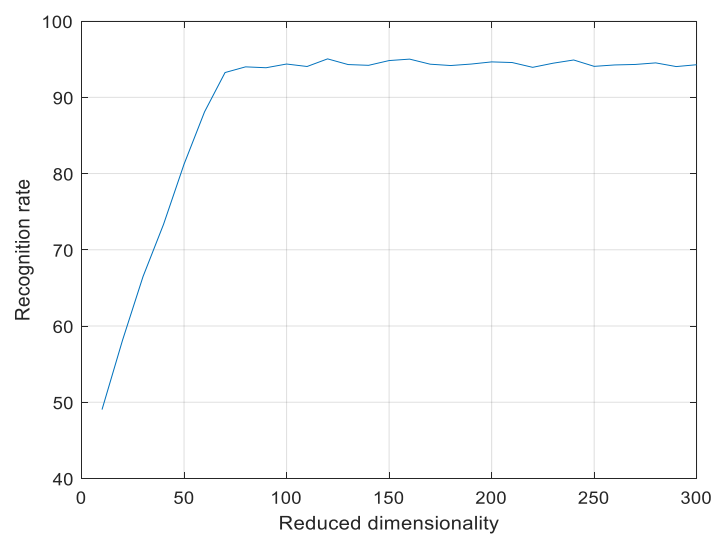

Fig. 6. Recognition rates of LDCCA versus the reduction dimensions.

For all methods, after obtaining eigenvectors, we choose $d$ eigenvectors corresponding to the $d$ largest eigenvalues whose sum dividing the total eigenvalues is no less than a predefined threshold. We set it as 0.95 in all experiments. Table 1 is the maximal average recognition accuracy with the corresponding standard deviations (std) of LDCCA and the comparison results of SATP, SIFT, LRP and CCA. From Table 1, it is found that LDCCA significantly outperforms the other algorithms in all the experimental cases.

Table 1. The Maximal Average Recognition Rates and the Corresponding Standard Deviations (Percent) of SATP, SIFT, LRP, CCA and LDCCA on the CASIA Dataset

\begin{tabular}{lccccc}
\hline Method & SATP & SIFT & LRP & CCA & LDCCA \\
\hline Recognition rate & $89.24 \pm 1.76$ & $90.17 \pm 1.69$ & $97.05 \pm 1.34$ & $95.47 \pm 1.41$ & $98.19 \pm 1.26$ \\
\hline
\end{tabular}

\subsection{Result Analysis}

Many palmprint identification methods have achieved better performance on the higher quality dataset. In fact, these methods often suffer from large intra-class variations on the CASIA dataset, because the palmprints 
of CASIA vary from pose, illumination, palm movement and other unconstrained conditions. From Table 1, it is seen that LDCCA has achieved the highest recognition rates. The main reason is that LDCCA makes use of the class information, local structure and correlation of the training data, including the left and right palmprints, to extract more discriminating and more robust features for personal identification. The recognition rates of LRP and CCA are higher than that of SATP and SIFT, which indicates that combining the left and right palmprints can improve the identification performance. The results of LDCCA and CCA verified that CCA is insufficient to find the nonlinear relationships between two sets of high-dimensional nonlinear features, while LDCCA can extract nonlinear features effectively by considering not only the correlations between the sample pairs but also the correlations between samples and their local neighborhoods.

\section{Conclusion}

In order to extract the useful features from left and right palmprints and achieve high recognition performance, a local discriminant canonical correlation analysis (LDCCA) based palmprint authentication method is presented by integrating left and right palmprints. This method takes the class information of samples into account when constructing the sample nearest neighbor graph, determines the weight by the distance measure between samples, and establishes the multiple weight correlation among left and right palmprints. By maximizing the weight correlation between paired samples and their nearest neighbors in the same class, it can also keep the local structure information of data while utilizing the class information of left and right palmprints. The experimental results on the CASIA database validate that LDCCA can extract more discriminant information in the low-dimensional projection feature subspace by more faithfully preserving local geometry and incorporating the class information and correlation of left and right palmprints. In the future, we will apply the proposed method to the multimodal biometric systems and investigate the fusion of multimodal biometric at different levels.

\section{Acknowledgment}

This work is partially supported by the Key Scientific Research Projects of Henan Higher Institutions Nos. 19B520029, 19B520028 and Henan Science and Technology Research Program Nos. 172102210512 and 172102210109.

\section{References}

[1] Abed, M. E., Giot, R., \& Hemery, B. (2012). Evaluation of biometric systems: A study of users' acceptance and satisfaction. International Journal of Biometrics, 4(3), 265-290.

[2] Leclercq, A. (2007). The perceptual evaluation of information systems using the construct of user satisfaction: Case study of a large french group. ACM SIGMIS Database, 38(2), 27-60.

[3] Lin, K., Wang, X., \& Cui, S. (2015). Heterogeneous feature fusion-based optimal face image acquisition in visual sensor network. Proceedings of IEEE Instrumentation and Measurement Technology Conference (pp. 1078-1083).

[4] Hemantha, K. K., \& Munaga, V. N. K. P. (2016). Palmprint identification using gabor and wide principal line Features. Procedia Computer Science, 93, 706-712.

[5] Imad, R., Romain, H., \& Gian, L. M. (2018). Palmprint recognition with an efficient data driven ensemble classifier. Pattern Recognition Letters.

[6] Wang, X., Lei, L., \& Wang, M. (2012). Palmprint verification based on 2D - Gabor wavelet and pulsecoupled neural network. Knowledge-Based Systems, 27, 451-455.

[7] Zhong, D., Du, X., \& Zhong, K. (2019). Decade progress of palmprint recognition: A brief survey. Neurocomputing, 328, 16-28 
[8] Morales, A., Kumar, A., \& Ferrer, M. A. (2016). Interdigital palm region for biometric identification. Computer Vision \& Image Understanding, 142(C), 125-133.

[9] Zhang, L., Li, L., \& Yang, A. (2017). Towards contactless palmprint recognition: A novel device, a new benchmark, and a collaborative representation based identification approach. Pattern Recognition, 69, 199-212.

[10] Feng, J., Wang, H., \& Li, Y. (2015). Palmprint Feature Extraction Method Based on Rotation-Invariance. Biometric Recognition Springer International Publishing, 215-223.

[11] Mouad, M. H. A., \& Gaikwad, A. T. (2016). Multimodal biometrics enhancement recognition system based on fusion of fingerprint and PalmPrint: A review. Global Journal of Computer Science and Technology: Graphics \& Vision, 16(2). Retrieved from https://www.researchgate.net/publication/310954018

[12] Lin, S., \& Tai, Y. (2016). A combination recognition method of palmprint and palm vein based on gray surface matching. Proceedings of International Congress on Image and Signal Processing (pp. 567-571).

[13] Kadri, F., Meraoumia, A., \& Bendjenna, H. (2016). Palmprint \& iris for a multibiometric authentication scheme using Log-Gabor filter response. Proceedings of IEEE International Conference on Information Technology for Organizations Development (pp. 1-5).

[14] Leng, L., Li, M, \& Kim, C. (2017). Dual-source discrimination power analysis for multi-instance contactless palmprint recognition. Multimedia Tools \& Applications, 76(1), 333-354.

[15] Charfi, N., Trichili, H., \& Alimi, A. M. (2017). Local invariant representation for multi-instance touchless palmprint identification. Proceedings of IEEE International Conference on Systems, Man, and Cybernetics (pp. 3522-3527).

[16] Wang, F., \& Zhang, D. (2013). A new locality-preserving canonical correlation analysis algorithm for multi-view dimensionality reduction. Neural Processing Letters, 37(2), 135-146.

[17] Peng, Y., Zhang, D., \& Zhang, J. (2010). A new canonical correlation analysis algorithm with local discrimination. Neural Processing Letters, 31(1), 1-15.

[18] Huang, X., Zhang, B., \& Qiao, H. (2017). Local discriminant canonical correlation analysis for supervised PolSAR image classification. IEEE Geoscience and Remote Sensing Letters, 14(11), 2102-2106.

[19] Mokni, R., Drira, H., \& Kherallah, M. (2017). Combining shape analysis and texture pattern for palmprint identification. Multimedia Tools and Applications, 76(22), 23981-24008.

[20] Qu, Z., \& Wang, Z. Y. (2011). The improved algorithm of scale invariant feature transform on palmprint recognition. Advanced Materials Research, 186, 565-569.

[21] Xu, Y., Fei, L., \& Zhang, D. (2015). Combining left and right palmprint images for more accurate personal identification. IEEE Transactions on Image Processing, 24(2), 549-559.

Shanwen Zhang was born in Shanxi Province, China. He received his B.S. degree in mathematics from Northwest University, China, in 1988. He received the M.S. degree in applied mathematics from Northwest Polytechnic University, China, in 1995. He received the Ph.D. degree in electromagnetic field and microwave from Air Force Engineering University, China, in 2001. He is a professor in the Xijing University, a visiting scholar in Department of Computer Science at Virginia Tech. His research area is machine learning and its application in data mining, including machine learning, leaf image processing, data reduction, data mining, feature selection, wavelet transforms, and their application in the plant disease recognition.

Qinging Zhang was born in Henan Province, China. She received her B.S. degree in electronic information engineering from Zhengzhou SIAS International University, China, in 2009. She received the M.S. degree in signal processing from Zhengzhou University, China, in 2013. Her research area is machine learning and its 
application in data mining, including machine learning, leaf image processing, data reduction, data mining, feature selection, wavelet transforms, and their application in the plant disease recognition.

Hao Yuan was born in Henan Province, China. He received his B.S. degree in electronic information engineering from Zhengzhou SIAS International University, China, in 2007. He received the M.S. degree in signal processing from Zhengzhou University, China, in 2018. His research area is machine learning and its application in data mining, including machine learning, leaf image processing, data reduction, data mining, feature selection, wavelet transforms, and their application in the plant disease recognition. 